\title{
EchoGéo
}

$8 \mid 2009$

Moyen-Orient : conflits et mobilités dans un espace mondialisé

\section{La sensibilité photographique du géographe}

\section{Anaïs Marshall}

\section{(2) OpenEdition}

Journals

Édition électronique

URL : https://journals.openedition.org/echogeo/11024

DOI : 10.4000/echogeo. 11024

ISSN : 1963-1197

Éditeur

Pôle de recherche pour l'organisation et la diffusion de l'information géographique (CNRS UMR 8586)

Référence électronique

Anaïs Marshall, «La sensibilité photographique du géographe », EchoGéo [En ligne], 8 | 2009, mis en ligne le 24 mars 2009, consulté le 31 juillet 2021. URL : http://journals.openedition.org/echogeo/ 11024 ; DOI : https://doi.org/10.4000/echogeo.11024

Ce document a été généré automatiquement le 31 juillet 2021.

EchoGéo est mis à disposition selon les termes de la licence Creative Commons Attribution - Pas d'Utilisation Commerciale - Pas de Modification 4.0 International (CC BY-NC-ND) 


\title{
La sensibilité photographique du géographe
}

\author{
Anaïs Marshall
}

1 Le géographe dispose, pour étudier le territoire, d'une palette d'outils les uns aussi pertinents que les autres. Même si la carte (ou plus récemment les systèmes d'information géographiques) reste l'élément qui est plus spontanément associé au territoire, la photographie est un vecteur très pertinent. L'approche photographique gagne à être prise en considération par la quantité d'informations utiles qu'elle permet de collecter, par la profondeur des perspectives qu'elle offre à l'interprétation et par la richesse des extrapolations subjectives qu'elle autorise. Bien évidemment toute photo n'a pas une portée scientifique, et tout géographe ne peut pas réaliser des photos appropriées sans un minimum de connaissance de cet outil.

2 Les différentes photographies présentées ici proposent de mettre en évidence l'information géographique visible et interprétable grâce à une identification et une description des éléments photographiés, puis à l'analyse scientifique des paysages et des sociétés. Cette série de clichés réalisée au Pérou entre septembre 2005 et juin 2008, permet d'exposer différents contrastes sociaux et environnementaux omniprésents dans le paysage et la société rurale de la côte désertique péruvienne. L'occasion aussi de mettre en avant la nécessité d'une mise en contexte et d'une indexation minimale permettant de replacer le cliché dans son contexte. Il y a un tel contrepoint, voulu parfois, entre l'expression visuelle et le fondement géographique ou social du cliché, qu'il arrive qu'on se méprenne sur la réalité à laquelle se réfère l'objet photographique. La subjectivité du géographe repose alors sur la sensibilité du photographe et sa capacité à saisir l'instant.

3 Dans quelle mesure la subjectivité du géographe-photographe permet-elle une interprétation et une analyse scientifique de la photographie?

4 Pour chaque photographie présentée, la légende est détaillée suivant cinq points :

- une description « à froid » de l'image ;

- des questions - non exhaustives - qui surgissent ou peuvent surgir à la vue du cliché ;

- une identification et description des éléments photographiés sans connaissance du terrain ; 
- une analyse complète grâce au travail de terrain (observations et entretiens) ;

- la méthode ou technique empirique appliquée lors de la prise de vue.

\section{A la recherche de l'eau}

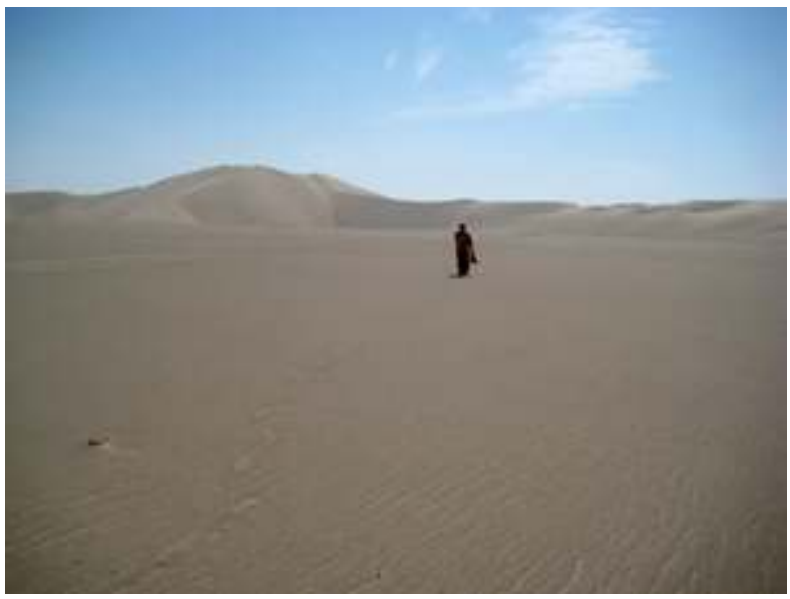
(ire que l'entrepreneur intéressé délimite le périmètre du terrain suivant les limites des autres terrains, et obtiendra son titre de propriété délivré par le ministère de l'Agriculture, si le lot est transformé en parcelle agricole fertile et rentable. Dans le cas contraire, le terrain redevient propriété de l'Etat.

11 Cette photo est celle d'un lot délimité récemment par une grande entreprise agroindustrielle péruvienne. La présence de ce gardien illustre l'appropriation et informe les autres acquéreurs susceptibles que le terrain est déjà occupé. Il a ainsi pour fonction de prévenir les éventuelles invasions de terre.

12 Lors de la visite de ce terrain, en août 2007, le gardien nous a conduit jusqu'à la limite nord du terrain, qui s'arrête aux pieds des dunes. Cette zone de dépression, qui n'apparait pas sur la photo, est une petite oasis formée de quelques algarrobos ${ }^{2}$. C'est ici que les propriétaires foreront les trois puits dans les jours qui viennent et, quelques mois après, cet espace désertique sera un champ d'asperges, d'oignons ou d'une autre culture vouée à l'exportation.

13 La prise a été réalisée lorsque l'acteur était à une distance suffisante pour montrer l'immensité du lieu, mais pas trop éloigné pour bien percevoir sa silhouette et son ombre. Le sujet n'est pas au centre pour donner une profondeur à l'image et mettre en avant l'étendue de son 
environnement. En effet, pour faciliter la lecture de la photo, le sujet n'est centré mais placé suivant la règle des tiers. Ce cadrage permet ainsi de répondre à la question: "Où va $t-i l$ ? vers les dunes ».

\section{Les asperges envahissent le désert}

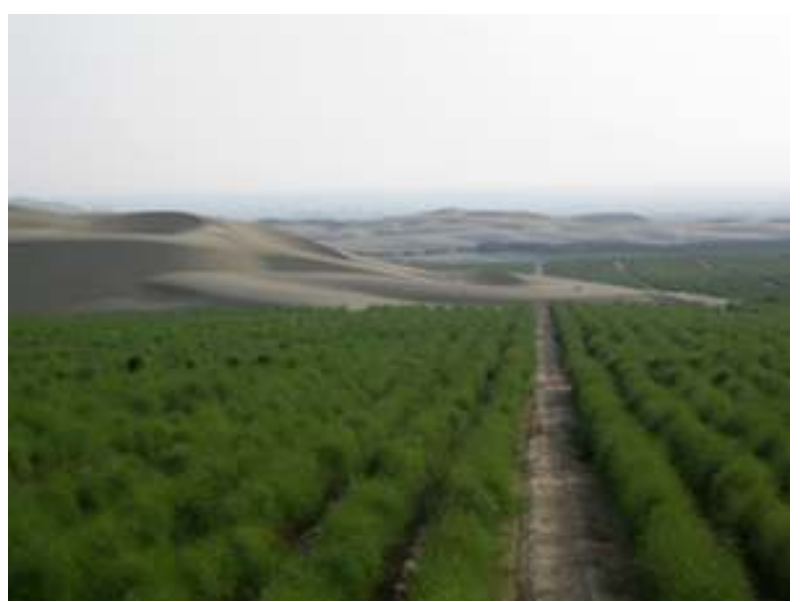

Au premier plan, une parcelle agricole et une dune qui la traverse.

Au deuxième plan, de nombreuses dunes, un semblant de ville et l'océan.

Quel type de culture ? Pour quels marchés?

La dune recouvre-t-elle le champ? Désertification? S'agit-il d'un recul des espaces cultivées recouvert par le sable du désert?

Les cultures sont-elles réalisées dans le désert ? L'extension des périmètres irrigués estelle réalisée sur les espaces désertiques?

Quelle est cette ville? Ses fonctions sont-elles industrielles, portuaires?

L'extension spatiale de l'exploitation et l'alignement des plantations permettent de déterminer que les parcelles sont travaillées de façon industrielle pour répondre à une importante demande commerciale.

Il est difficile de déterminer si la dune avance sur les cultures, ou si le champ s'étend sur le désert.

Il est aussi difficile d'interpréter le dernier plan qui laisse entrevoir une ville.

Cette photo a été prise dans l'interfluve entre les vallées de Virú et de Moche, près de la ville de Trujillo, dans le Nord du Pérou. Les vallées de Chao, Virú, Moche et Chicama sont comprises dans l'aire d'influence d'un grand projet étatique: le projet Chavimochic - PECH. Ce projet a consisté à dévier les eaux d'un bassin versant, le Santa, vers les quatre vallées grâce à un canal de 285 kilomètres qui traverse le désert côtier. Cette déviation avait deux objectifs agricoles: l'apport d'une ressource en eau constante aux vallées qui souffraient de sécheresse plusieurs mois par an, et l'extension de la frontière agricole dans les interfluves désertiques.

Ce cliché met ainsi en évidence l'extension des périmètres irrigués dans le désert. Toutes les zones planes sont plantées de diverses productions, ici d'asperges, destinées à l'exportation. Ce sont ainsi les périmètres irrigués qui sont gagnés sur les espaces désertiques. Au deuxième plan, on aperçoit le port de Salaverry d'où partent de 
nombreux containers à destination des Etats-Unis. Pour des destinations plus lointaines comme l'Europe et le Japon, les asperges fraîches seront envoyées par avion au départ de l'aéroport de Lima.

La photo a été prise en limite de parcelle pour valoriser visuellement l'étendue des périmètres irrigués. Les lignes des cultures de l'asperge sont perpendiculaires à l'axe de la dune afin de mettre en avant le contraste et la confrontation des deux environnements.

Le choix a été fait de mettre en dernier plan l'océan et non le piémont pour mettre en évidence l'exportation des productions. Si le piémont apparaissait en arrière plan, les thématiques de ressources en eau auraient été mises en avant.

\section{Bourg à aménagement spontané : invasion puis implantation des populations migrantes}

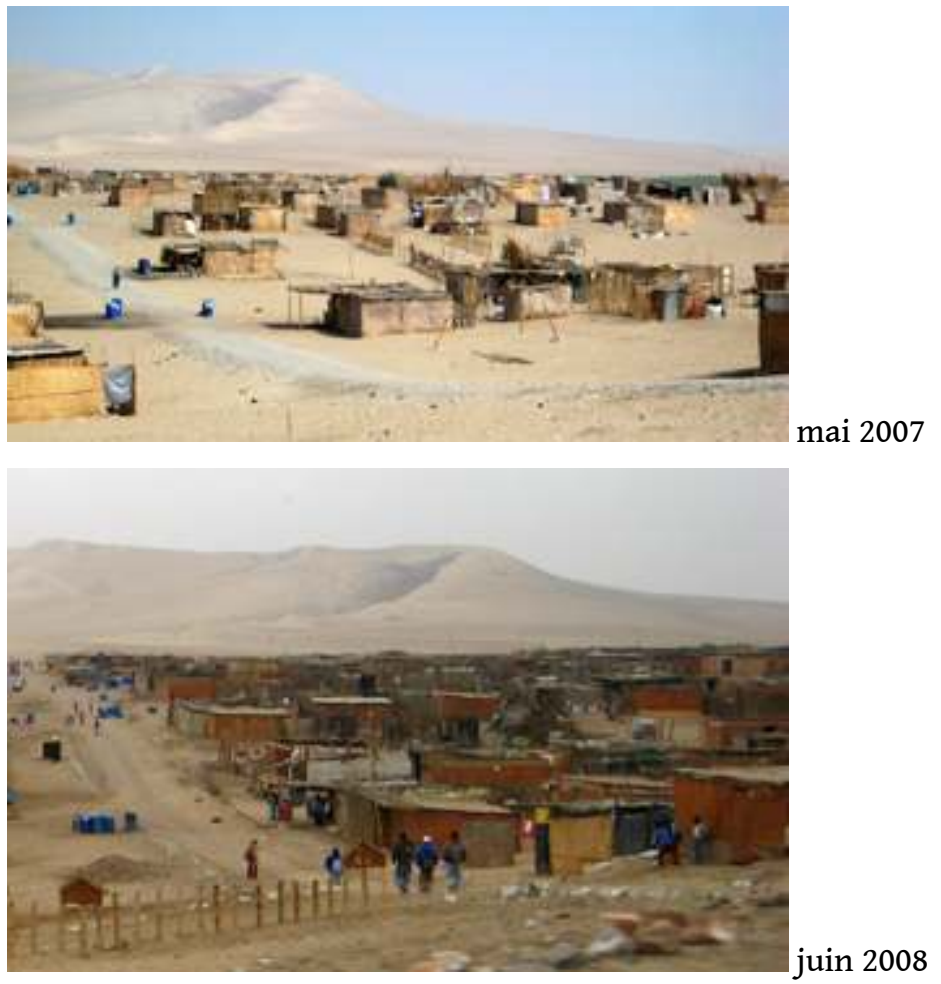

Deux photos, un même lieu. La première photo présente quelques baraques construites en paillasse dans le désert. Un chemin traverse les constructions. La deuxième photo présente le même lieu avec davantage de constructions. Les maisons de sont plus en paillasse mais en planche de contreplaqué.

Où sommes-nous? Est ce un bourg? Pourquoi cette installation "urbaine » dans le désert? Pourquoi cette extension et cette densification?

Sur les deux clichés, la présence d'individus sur le chemin et devant les maisons, et les bidons bleus (poubelle ou réserve d'eau) témoignent du caractère résidentiel de ce bourg. La juxtaposition des deux images met en avant l'évolution et les dynamiques spatiales du bourg. La diachronie est une méthode d'analyse très utilisée par les géographes, notamment à partir de photos aériennes ou d'images satellite. Ces deux photos obliques prises à un an d'écart traduisent la rapidité de la dynamique d'extension et de densification de la zone habitée. 

panaméricaine, avant la vallée d'Ica. Suite à l'implantation des entreprises agroindustrielles au sein du désert côtier et à l'extension des périmètres irrigués, les offres d'emplois ont considérablement augmenté. La population locale ne suffit plus à satisfaire la demande, et les migrations nationales se développent, les travailleurs souhaitant être embauchés comme ouvriers agricoles. Face à l'absence de politiques publiques d'aménagement du territoire, les migrants s'installent sur des terrains laissés vacants. Dans un premier temps plusieurs dizaines de famille envahissent les terres, souvent la nuit, en installant des maisons de fortune. Ensuite, au fur et à mesure des rentrées d'argent, les maisons sont consolidées. clichés, un tremblement de terre a eu lieu. Comme l'Etat n'intervenait pas pour l'aménagement de ce nouveau centre peuplé, les habitants, bien que peu affectés par le séisme, ont fait appel aux institutions internationales. Ils ont ainsi bénéficié de l'aide internationale, destinée originellement aux populations affectées par le désastre naturel, pour obtenir des maisons en bois.

Les deux clichés ont été pris selon un point de vue quasiment identique pour permettre une comparaison aisée et faciliter la perception de la dynamique des changements. La faible luminosité de la deuxième image appuie l'impression de concentration des habitats.

\section{Francisca et sa fille : productrices de pastèques à Virú}

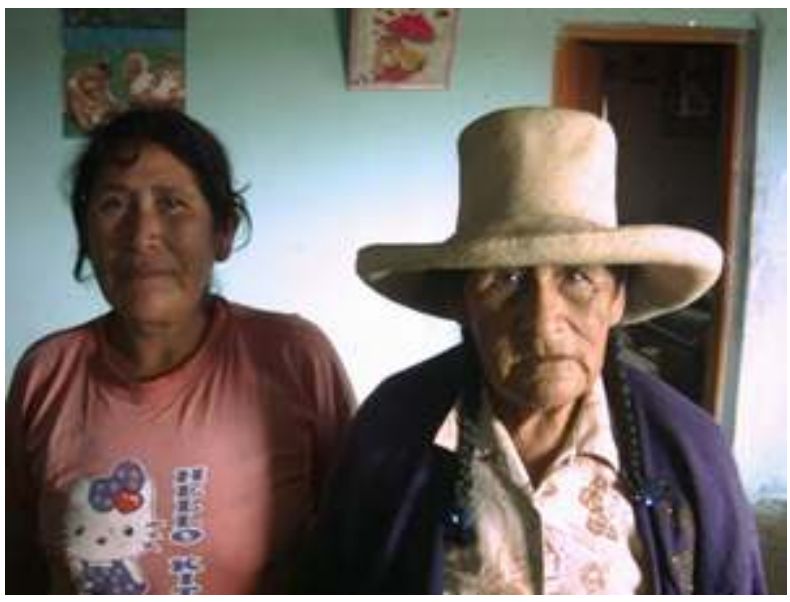

Portraits de deux femmes dans une maison.

Qui sont-elles? Que font-elles? Où sont-elles?

De nombreux détails peuvent être analysés. La femme la plus âgée porte un chapeau typique des paysans de la côte (?), elle est coiffée comme les femmes originaires des Andes. La femme plus jeune porte un tee-shirt de marque occidentale, et plus spécifiquement japonaise. Ce cliché met en exergue les différences intergénérationnelles.

Derrière les deux femmes, on entrevoit la maison: mur peint en vert, une porte qui laisse entrevoir une autre pièce et deux cadres. Le premier est une image qui représente une famille occidentale pique-niquant dans un jardin, le deuxième est un tissu brodé qui représente un personnage de conte pour enfants. Les cadres présentent 
le contraste entre la réalité et l'imaginaire. L'intérieur de cette maison est similaire à celui des maisons rurales typiques construites en adobe.

La photo présente Francisca et sa fille. Elles sont productrices de pastèques. Leurs terres se situent dans la vallée de Virú, dans le département de La Libertad sur la côte nord du Pérou. Il y a 60 ans, Francisca est partie de Cajabamba - situé sur le versant amazonien des Andes - avec sa famille. Ils ont défriché des espaces de forêts sèches pour les transformées en terres cultivables. Elle gère à présent l'entreprise familiale et travaille avec ses enfants et petits-enfants. Elle vend sa production directement sur sa parcelle à des commerçants qui la revendent ensuite sur les marchés locaux ou nationaux.

La photo a une luminosité particulière qui met en avant Francisca, contrairement à sa fille qui est moins éclairée et en retrait. Ce cadre et cette disposition met en exergue le rôle et l'importance de la mère au sein de cette famille.

\section{Le paiement de l'eau : intimité publique}

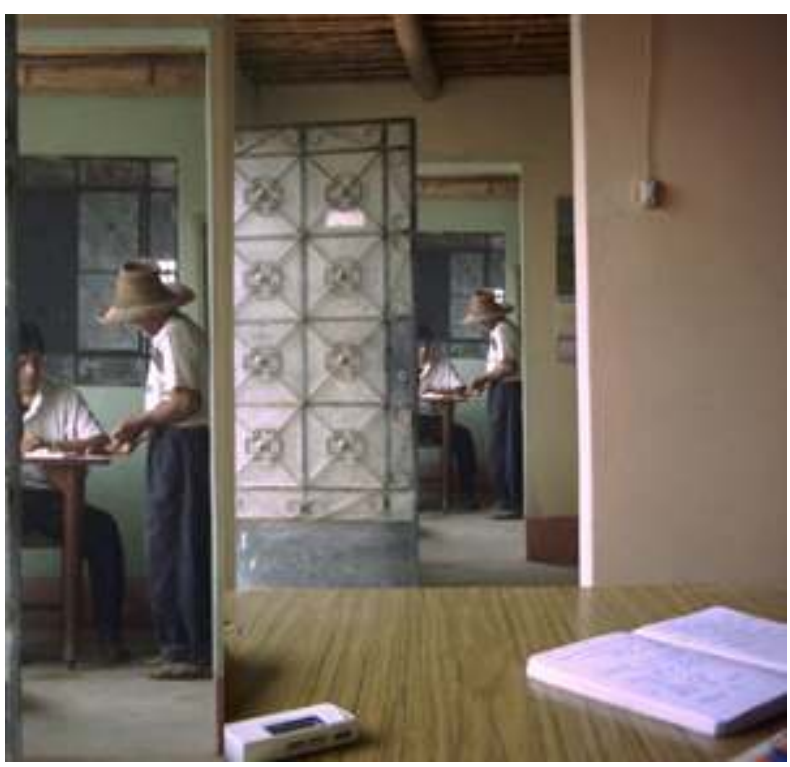

Deux photos jointes.

A gauche, photo à plans divers : premier plan un cahier ouvert et un dictaphone, deuxième plan une porte et des pans de murs, dernier plan deux hommes discutant : l'un assis l'autre debout.

Qui sont-ils ? Où sont-ils ? Quels rôles et quelles relations ont-ils ?

Pourquoi cet assemblage de photos?

L'homme debout semble de condition modeste par sa tenue vestimentaire, notamment ses sandales et son chapeau. Il présente quelque chose à l'homme assis derrière une table. Ce dernier semble être un notable par sa position (le bureau est une représentation symbolique du pouvoir).

A droite, l'image est un zoom du dernier plan.

Ces clichés présentent un agriculteur qui vient "acheter son eau ». Dans les vallées côtières du Pérou, l'Etat est chargée de la distribution de l'eau et de l'entretien des canaux d'irrigation. A fréquence régulière qui varie en fonction du type de cultures, 
l'agriculteur achète un nombre d'heures durant lesquelles les vannes sont ouvertes pour que l'eau des canaux gravite dans les sillons de ses parcelles.

La succession de plans met en exergue le contraste entre le caractère administratif et public d'une formalité administrative banale et la facette intimiste des relations de pouvoir qui sousentendent le système foncier du désert côtier péruvien.

L'objectivité voulue du géographe, à travers ses productions scientifiques (cartes, entretiens, photographies), est essentielle. Il est couramment mentionné que l'on peut faire dire ce que l'on souhaite à une carte, il en va de même pour une photographie. L'angle de vue, le cadrage, la couleur, la luminosité de la prise, le sujet, l'assemblage, etc. influencent l'interprétation. Chouvy (2008) précise que la photo ne dit pas systématiquement grand-chose du lieu et du sujet, et qu'elle laisse donc une grande part à l'interprétation. Il revient ainsi au chercheur de proposer une représentation photographique neutre et fidèle de la réalité, réduisant la subjectivité de la prise de vue et menant à une interprétation univoque.

\section{BIBLIOGRAPHIE}

Angotti Salgueiro H. 2001, L'image dans l'œuvre de Pierre Monbeig. Intergéo Bulletin 1-2001. L'image au cœur de la géographie PRODIG-CNRS. p. 77-84.

Bertrand F., Goeldner-Gianella L., Husberg T., 2004. Paysages vus du ciel et fiches-images pédagogiques. Landscapes from above and educational files.Numéro spécial de Photo interprétation, ISSN 0031-8523, vol. 39, n 3-4.

Chatelain, P. 1989. Quelques réflexions sur les rapports de la géographie à l'image, Strates [http://strates.revues.org/document4042.html], Numéro 4-1989, Dossier images réfléchies. Paroles d'un paysan révolutionnaire

Chouvy P-A., 2008. Tropisme géographique et tropisme photographique, EspacesTemps.net, Mensuelles, http://espacestemps.net/document5603.html

Huagonie G. 1998. Du bon usage de la photographie de paysage en géographie: comment faire parler l'image. http://innovalo.scola.ac-paris.fr/Innovatio/innovatio4/imagegeo.htm

Luginbühl Y., 2001. L'Observatoire Photographique du paysage. Intergéo Bulletin 1-2001. L'image au cœur de la géographie PRODIG-CNRS. p. 63-68.

Ormaux S., 2001. Ceci est-il un paysage ? Ou quelques propositions pour un nouvel usage de la photographie et géographie. Intergéo Bulletin 1-2001. L'image au cœur de la géographie PRODIG CNRS. p. 9-15.

Tallet B., 2001. Les parcs arborés révélateurs des logiques paysannes en Afrique Noire. Voir pour comprendre l'image comme support de l'analyse des paysages. Intergéo Bulletin 1-2001. L'image au cœur de la géographie PRODIG-CNRS, p. 29-32

Revue : La géographie (notamment n 4, Guerres et conflits) 


\section{NOTES}

1. Le terme vierge est entre guillemets car les de nombreuses zones désertiques appartenaient à des communautés paysannes. (cf. Marshall, thèse en cours)

2. Algarrobo (Acacia macracantha) est un arbre typique du désert péruvien qui compose les forêts sèches.

\section{RÉSUMÉS}

Pour l'analyse des territoires, le géographe possède de nombreux outils. L'approche photographique doit être considérée d'avantage pour les perspectives de recherche qu'elle offre. Dans quelle mesure la sensibilité photographique du géographe permet une interprétation objective de l'analyse scientifique? Six photographies décrites, puis interprétées grâce à un travail de terrain proposent de répondre à cette question.

For study territories, geographers Scientifics have various tools. The photographic approach has to be more considerate for the research perspectives offered. We can ask then if the photographic sensibility of the geographer is really allow an objective interpretation and a scientific analysis. To answer, six pictures of Peru have been first described and then analysed with the help of the fieldwork. Finally the geographer-photographer has to propose an objective picture, neutral and conform to the reality and easily interpreted by everyone.

\section{INDEX}

Mots-clés : sensibilité photographique, interprétation subjective, analyse visuelle, Pérou, prise de vue

Keywords : photographic sensibility, interpretation, visual analysis, Peru, method

\section{AUTEUR}

\section{ANAÏS MARSHALL}

Anaïs Marshall (anaismarshall@gmail.com) est doctorante à l'Université de Paris 1 PanthéonSorbonne et membre de l'UMR CNRS-PRODIG. 